\title{
Analysis of Persian Oak (Quercus Brantii Lindl)'s Spectral- Temporal Behaviour Under the Stresses of Water Deficiency and Dust Particles
}

\author{
Ali Darvishi Boloorani ${ }^{1, *}$, Soghra Ranjbareslamloo ${ }^{1}$, Saham Mirzaie ${ }^{1}$, Hossein Ali Bahrami ${ }^{2}$, Fardin Mirzapour ${ }^{3}$, \\ and Nadia Abbaszadeh Tehrani ${ }^{4}$ \\ ${ }^{1}$ Department of RS\&GIS, Faculty of Geography, University of Tehran, Iran \\ ${ }^{2}$ Department of Soil, Faculty of Agriculture, Tarbiat Modares University, Iran \\ ${ }^{3}$ Department of Electrical Engineering-Sadra Institute of Higher Education, Iran \\ ${ }^{4}$ Aerospace Research Institute, Ministry of Science, Research and Technology of Iran, Iran
}

\begin{abstract}
Persian oak (Quercus Brantii Lindl) is the most abundant tree species in Zagros mountain range that is affected by western dust storms and harsh droughts in recent years. The lack of spectral-temporal information about these trees has caused limitations on the usage of remote sensing images to make a synoptic estimation of damages caused by dust storms and drought. The objective of this research is to analyse the spectral-temporal behaviour of Persian oak under the stress of water deficiency and dust storms. The aim is to improve the competitive abilities of experts on modelling the stress of water deficiency and dust storms on trees using remote sensing images. For the purpose of this experimental investigation, a greenhouse laboratory has been built. Analysis of the experiments was carried out using 54 two-year-old oak tree seedlings; Fieldspec-3-ADD; and wind tunnel at the greenhouse of the faculty of agriculture of the Tarbiat Modares University in 2016. Results show that the water stress could be modelled much better using geometrical indices extracted from continuum removed spectrum. Area and the depth were best indices. Water stress has been modelled better that dust stress.
\end{abstract}

\section{Introduction}

Drought is one of the most threatening natural phenomena for Persian oaks (Quercus Brantii Lindl) native to Iran, Iraq, Syria, Turkey, and West Asia. Though many factors have been mentioned for oak decline, drought and water deficiency are playing the main role for this phenomena in Zagros mountain range. On the other hand these forests are located in the West Asian dust corridor that starts from Syrian deserts and ends at the Indian Ocean. These windblown dust particles are another main stressor for trees in the region. Several remote sensing and spectrometry methodologies and techniques have been developed for modelling the behaviour of different trees under different stresses [1].

Imaging and non-imaging hyperspectral data are used for measuring the chemical-physical properties of plants and the application of field spectrometry offers opportunities to identify biochemical characteristics of plants $[2,3]$. Plant stresses have signatures in internal and external factors of leaf cellules [4] and any changes in these factors will have spectral signature on plant reflectance [5]. Measuring these signatures by spectrometers will help to model the kind and level of stresses. Environmental stresses have a variety of types and levels [6], within them, dust particles are multiinfluential stressors that impact on physical, chemical and physiological characteristics of leaves, dry biomass $[7,8]$, chlorophyll [9], transpiration [10] and leaf surface temperature [11].

Remote sensing methods for plant parameters estimation are mainly based on statistical and physical models along with their advantage and disadvantages. Sensitivity analysis of plant indices using single and multiple regression analysis are very common in plant stress modelling with remote sensing imagery. Ač et al., [12] investigated the fluorescence chlorophyll potential for water, heat, and nitrogen stresses in leaf and canopy cover levels. Results showed that there is a meaningful relation between water stress and the rate of fluorescence signal in red and NIR spectrum. Hyperspectral images are also sowing high performance in measuring plant water stress [13], nutrient deficiency [14, 15].

The goal of this experimental research is to analyse Persian oak's spectral-temporal behaviour under the stress of water deficiency and dust storms.

\section{Material and Methodology}

Two-year-old oak seedlings within plastic pots are used. The experiment was carried out using a total of 58 seedlings planted in March 2014 (before blossom bud season). The water and dust stresses were carried out in a plastic greenhouse. During the stresses, the greenhouse temperature, humidity, and air condition are controlled and measured. Soil samples from the sources of dust

\footnotetext{
* Corresponding author: ali.darvishi@ut.ac.ir
} 
storms are collected and using a portable wind tunnel, dust storms were simulated. The greenhouse is divided into four sections. Three levels of dust concentration, i.e. 350, 750 , and $1500 \mu \mathrm{g} / \mathrm{m}^{3}$ (measured using 176000A Microdust Pro Dust Monitor). Within three sections of the greenhouse dust storms were simulated using wind tunnel, and one section was left with no dust for control samples. The greenhouse drainage system is installed and adapted in the four sections for trees water stress, i.e. 100, 40 and $20 \%$ of field capacity (measured using Time Domain Reflectometer, TDR).

The 45 seedlings from the 58 samples were put into three sections, i.e. 15 for each. Water stress was induced on seedlings by means of changes in irrigation practices, i.e. well-watered $(100 \%$ field capacity), medium water deficiency condition ( $40 \%$ field capacity), and severe water deficiency condition ( $20 \%$ field capacity) treatments. In this experimental work 4 seedlings were totally dried and lost their leaves, therefore we neglect them in the spectrometry phase of the work. One sample from each section at the end of stress experiments are shown in Fig. 1.

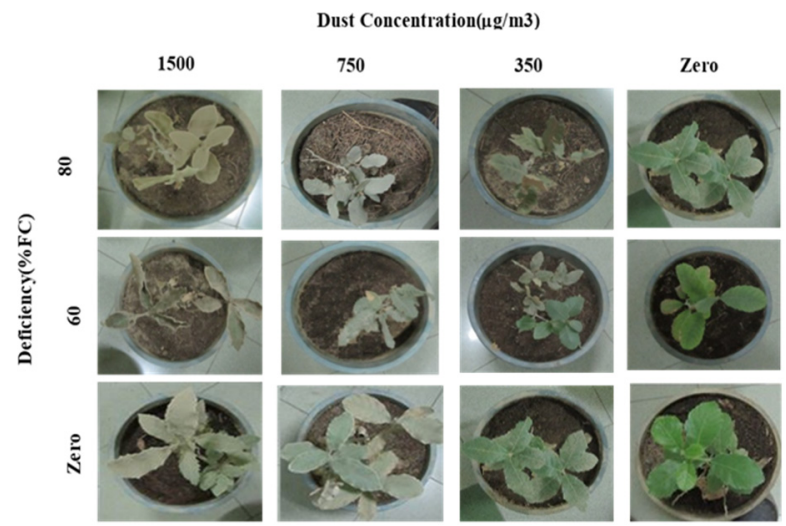

Figure 1. Impacts of different dust concentration and water levels.

After finishing the experimental phase in the greenhouse, the samples were moved into a darkroom of the spectrometry laboratory. ASD FieldSpec 3 spectroradiometer was used for leaves level spectral measurement. We used plant probes for measurement. In order to obtain a proper reflectance, 8 spectral measurements for each pot were done. Instrument calibration was carried out after each 5 measurements. Immediately after spectrometry, we measured the amount of chlorophyll present in three leaves from each sample using SPAD. The average of these three measurements is used for the next analysis.

In order to measure the leaf water contents, they were weighted before and after drying process. Leaves were dried at $70^{\circ} \mathrm{C}$ for 24 hours through oven drying. Leave water contents were calculated using equation [16]:

$$
M C=100 \frac{F-D}{F} .
$$

Here, $\mathrm{F}$ is fresh leaf weight and $\mathrm{D}$ is dried leaf weight. For each sample the standard deviation of the 8 individual measurements was calculated and the result shows that the region between $350-400$ and $2400-2500 \mathrm{~nm}$ has \pm 2 standard deviations to the mean of each sample. Therefore, the region $400-2400 \mathrm{~nm}$ was used for next steps of processing. In order to reduce the instrument and illumination errors from the spectrals, the Savitzky and Golay smoothing filter (frame size of 15) and a polynomial of degree two are adapted to smooth all spectrals. After smoothing the arithmetic mean of these eight measurements was used for the next steps of analysis. In the next step, the noisy and useless data were eliminated by using Principal Component Analysis (PCA) and visual investigations. Finally, the spectral of 54 Persian oak samples are used for measuring water and dust stresses.

Absorption Features (AFs) are those features in spectra that are related to the chemical-physical characteristics of objects. Spectral measurements contain both AFs and continuum data. After continuum removal using equation (2) $[17,18]$, the four geometric characteristics of AFs were calculated, i.e. width, depth, asymmetry, and skewness:

$$
R_{C}(\lambda)=\frac{R_{O}(\lambda)}{R_{L}(\lambda)} .
$$

Here, $R_{C}$ is the continuum removed spectra, $R_{o}$ is the original spectra, and $R_{L}$ is the continuum curve. In Fig. 2 selected AFs are represented for reflectance and continuum removal. Also geometrical indices for continuum removal illustrated.
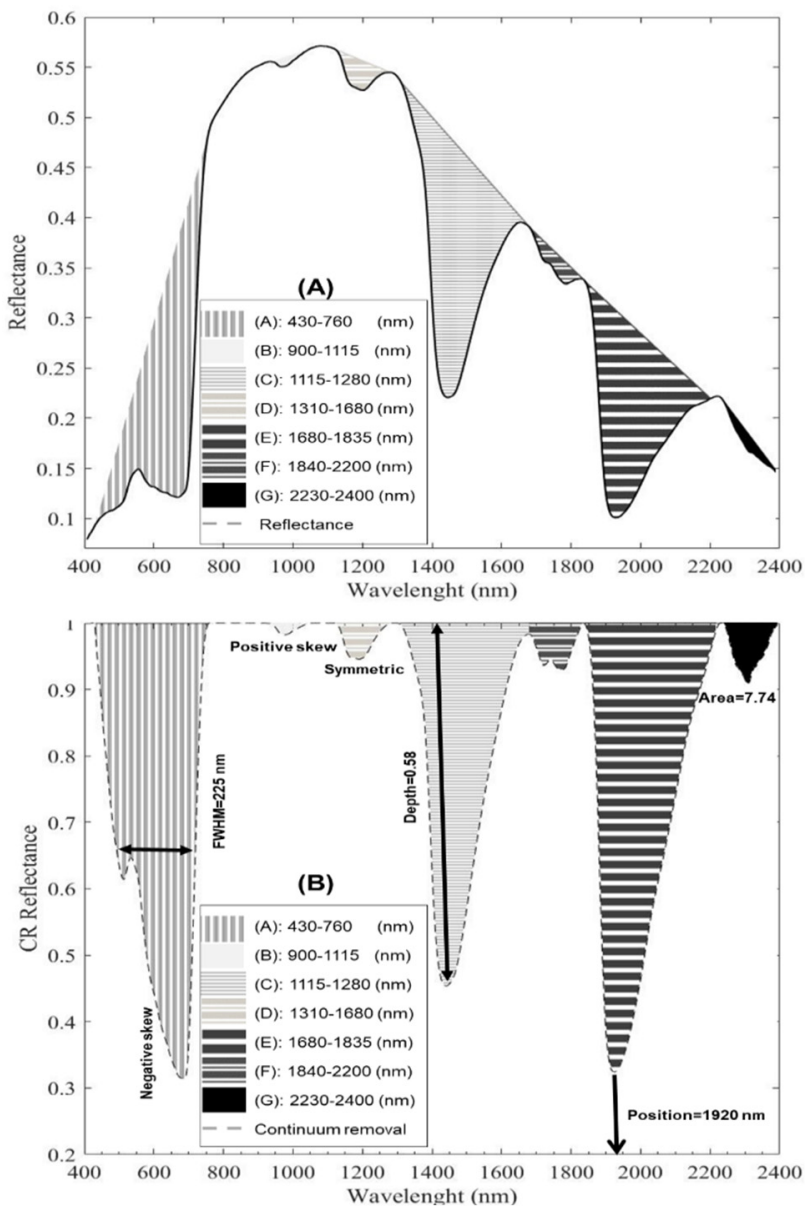

Figure 2. A) Spectral reflectance and selected AFs for study, B) continuum removal of reflectance and geometrical indices. 


\section{Results and discussion}

Results of the experiments that were carried out using 54 two-year-old oak tree seedlings; Fieldspec-3-ADD; and wind tunnel at the greenhouse. Water stress given to the seedlings by changes in irrigation practices, i.e. 100, 40 and $20 \%$ of the trees water requirements. Dust particle stress also given in three different dust particle concentrations, i.e. 350,750 and $1500 \mu \mathrm{g} / \mathrm{m}^{3}$ using portable wind tunnel in greenhouse. The spectrometry experiments were carried out at leaf level dark room. All samples had been handled uniformly.

Mean of leaf chlorophyll content for different level of each stress samples illustrate in the Fig. 3. Increasing the level of dust and water stress lead to reduced chlorophyll concentration of leafs.

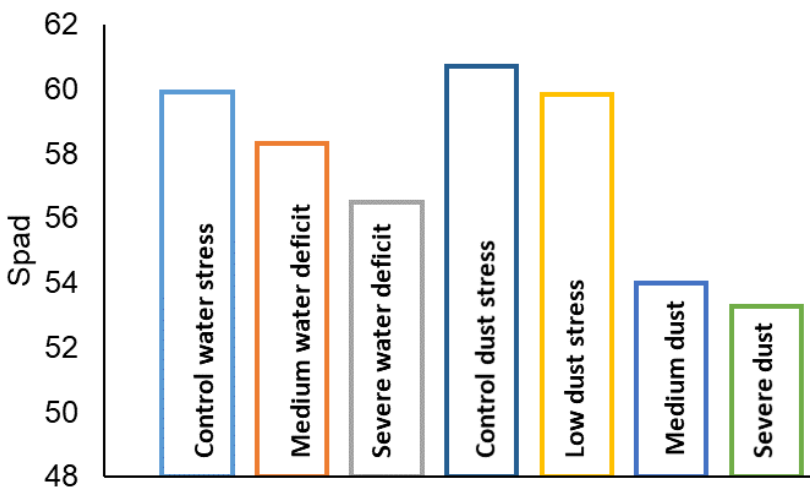

Figure 3. Summary statistics of leaf chlorophyll content for different water and dust stress levels sample.

The mean of leaf moisture for different levels of each stress sample are illustrated in the Fig. 4. Increasing the level of water stress leads to a large reduction of leaf moisture, whereas, in low level dust concentration $\left(350 \mu \mathrm{g} / \mathrm{m}^{3}\right)$ leaf moisture shows a little increase. For medium and severe level of dust leaf moisture shows somewhat lower value.

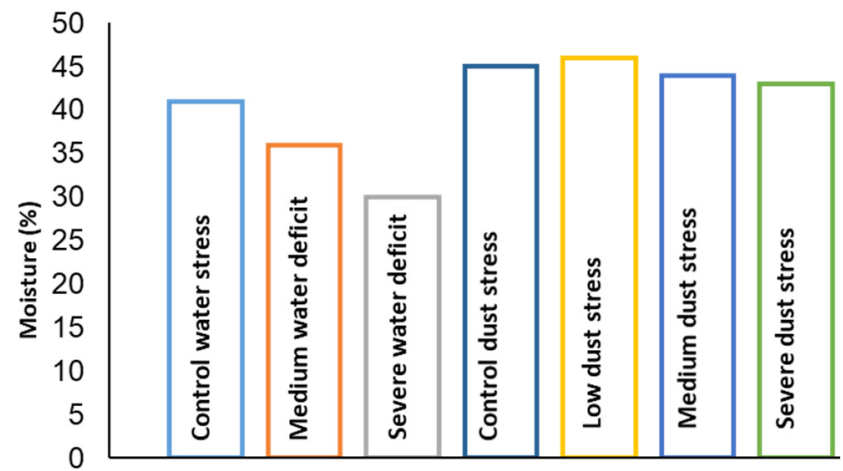

Figure 4. Summary statistics of leaf moisture content for different water and dust stress levels sample.

Area, depth, asymmetry, FWHM (width) and position are 5 indices that extracted for 7 main AFs of oak seedlings continuum removed spectrum. Correlation and RMSE of these indices and water and dust stress levels are shown in Tab. 1 and Tab. 2, respectively. Indices with high correlation have grey colour background.
Negative correlation between area index and water stress, means that with increasing level of water stress the AFs (A, D, F) area is reduced, except G. Like area we have negative correlation between all of the AFs for depth index, except G. It means that, with increasing level of stress the depth of all of AFs is reduced, while increasing the level of water stress increase the depth and area of AF G. For asymmetry just AFs $\mathrm{C}$ and $\mathrm{G}$ have a high correlation. It shows that with increasing level of stress, we will have positive skewness (Right skew) for both of them. Positive correlation is found between AF E width and water stress level. AFs $\mathrm{A}$ and $\mathrm{C}$ have positive correlation with water stress. It means that with increasing level of water stress the position of deepest band of AFs will be shifted to higher wavelength. While, for AF E the deepest band is shifted to lower wavelength by increasing the stress level (Tab. 1).

Table 1. Correlation and RMSE between geometrical indices and water stress levels.

\begin{tabular}{|c|c|c|c|c|c|c|}
\hline \multirow[b]{2}{*}{ 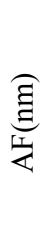 } & & \multicolumn{5}{|c|}{ Extracted indices } \\
\hline & & 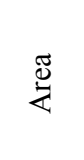 & $\begin{array}{l}\bar{\Xi} \\
\stackrel{\overline{0}}{0}\end{array}$ & 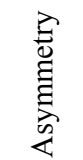 & $\sum_{I}^{\sum_{I}^{1}}$ & 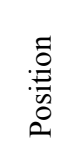 \\
\hline \multirow{2}{*}{ A } & $R$ & -0.78 & -0.83 & 0.24 & -0.44 & 0.63 \\
\hline & $R M S E$ & 0.23 & 0.2 & 0.35 & 0.3 & 0.30 \\
\hline \multirow{2}{*}{ B } & $R$ & -0.28 & -0.34 & 0.31 & -0.15 & 0.37 \\
\hline & $R M S E$ & 0.35 & 0.34 & 0.35 & 0.36 & 0.35 \\
\hline \multirow{2}{*}{$\mathrm{C}$} & $R$ & -0.1 & -0.55 & 0.74 & -0.22 & 0.73 \\
\hline & $R M S E$ & 0.37 & 0.36 & 0.24 & 0.36 & 0.25 \\
\hline \multirow{2}{*}{$\mathrm{D}$} & $R$ & -0.66 & -0.72 & 0.225 & 0.17 & 0.06 \\
\hline & $R M S E$ & 0.27 & 0.25 & 0.36 & 0.36 & 0.38 \\
\hline \multirow{2}{*}{$\mathrm{E}$} & $R$ & 0.37 & 0.1 & 0.31 & 0.61 & -0.56 \\
\hline & $R M S E$ & 0.34 & 0.37 & 0.35 & 0.29 & 0.30 \\
\hline \multirow{2}{*}{ F } & $R$ & -0.83 & -0.84 & 0.07 & -0.38 & -0.19 \\
\hline & $R M S E$ & 0.2 & 0.2 & 0.37 & 0.34 & 0.368 \\
\hline \multirow{2}{*}{$\mathrm{G}$} & $R$ & 0.54 & 0.47 & 0.5 & 0.46 & 0.44 \\
\hline & $R M S E$ & 0.31 & 0.32 & 0.32 & 0.32 & 0.33 \\
\hline
\end{tabular}

Area and depth indices have negative correlation with water stress. It means that with increasing the level of dust stress all of the AFs (A, B, C, D, E, F, G) area and depth will be reduced. Asymmetry and width have no meaningful correlation with dust stress. Deepest band of AF D will be shift to higher wavelength with increasing the level of dust concentration (Tab. 2).

\section{Conclusion}

The main conclusions of this experimental research are: (i) Leaf chlorophyll concentration of oak seedling is reduced by increasing the level of dust and water stress. (ii) Leaf moisture concentration of oak seedling is reduced by increasing level of water stress, while for dust stress we have not clear pattern. (iii) Both stresses in leaf can be recognized using geometrical indices extracted from continuum removal; (iiii) area and depth indices are the good geometric indices for most of the AFs and both stresses. In conclusion, the research shows that for water stress we have better and reliable result, but we are faced 
with an uncertain impact of dust particles on the plants and more investigations on that are needed in the future.

Table 2. Correlation and RMSE between geometrical indices and dust stress levels.

\begin{tabular}{|c|c|c|c|c|c|c|}
\hline \multirow[b]{2}{*}{ 氞 } & & \multicolumn{5}{|c|}{ Extracted indices } \\
\hline & & 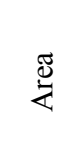 & 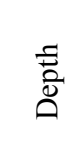 & 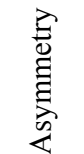 & $\sum_{i}^{\sum_{1}}$ & 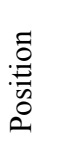 \\
\hline \multirow{2}{*}{ A } & $R$ & -0.52 & -0.51 & 0.02 & -0.37 & 0.22 \\
\hline & $R M S E$ & 0.53 & 0.53 & 0.62 & 0.57 & 0.61 \\
\hline \multirow{2}{*}{$\mathrm{B}$} & $R$ & -0.52 & -0.59 & -0.20 & -0.02 & 0.12 \\
\hline & $R M S E$ & 0.53 & 0.31 & 0.61 & 0.62 & 0.62 \\
\hline \multirow{2}{*}{$\mathrm{C}$} & $R$ & -0.48 & -0.50 & -0.07 & 0.07 & -0.06 \\
\hline & $R M S E$ & 0.54 & 0.54 & 0.62 & 0.63 & 0.63 \\
\hline \multirow[b]{2}{*}{$\mathrm{D}$} & $R$ & -0.54 & -0.55 & 0.46 & -0.28 & 0.42 \\
\hline & $R M S E$ & 0.52 & 0.52 & 0.55 & 0.60 & 0.57 \\
\hline \multirow{2}{*}{$\mathrm{E}$} & $R$ & -0.41 & -0.49 & -0.20 & -0.24 & 0.18 \\
\hline & $R M S E$ & 0.57 & 0.54 & 0.61 & 0.60 & 0.61 \\
\hline \multirow{2}{*}{$\mathrm{F}$} & $R$ & -0.51 & -0.44 & 0.44 & -0.26 & 0.10 \\
\hline & $R M S E$ & 0.54 & 0.60 & 0.63 & 0.54 & 0.62 \\
\hline \multirow{2}{*}{ G } & $R$ & -0.28 & -0.27 & 0.08 & -0.04 & 0.05 \\
\hline & $R M S E$ & 0.60 & 0.60 & 0.62 & 0.60 & 0.63 \\
\hline
\end{tabular}

\section{References}

1. G.P. Asner, Remote Sens. Environ. 64, 234 (1997)

2. G.P. Asner, C.A. Wessman, C.A. Bateson, and J.L. Privette, Remote Sens. Environ. 74, 69-84 (2000)

3. R. Darvishzadeh, A. Skidmore, M. Schlerf, and C. Atzberger, Remote Sens. Environ. 112, 2592-2604 (2008)

4. R.D. Jackson, Ann. Rev. Phytopathol. 24, 265-287 (1986)

5. H.C. Stimson, D.D. Breshears, S.L. Ustin, S.C. Kefauver, Remote Sens. Environ. 96, 108-118 (2005)

6. N. Spieß, M. Oufir, I. Matusikova, M. Stierschneider, D. Kopecky, A. Homolka, K. Burg, S. Fluch, J.F. Hausman, E. Wilhelm, Environ. Exper. Bot. 77, 117$126(2012)$

7. A.M. Farmer, Environ. Pollut. 79, 63-75 (1993)

8. E. Darley, J. Air Pollut. Control Assoc. 16, 145-150 (1966)

9. D.V. Armbrust, Agron. J. 78, 1078-1081 (1986)

10. D.W. Eueling, Ann. Appl. Biol. 64, 139-151(1969)

11. B.M. Eller, Environ. Pollut. 13, 99-107 (1977)

12. A. Ač, Z. Malenovský, J. Olejníčková, A. Gallé, U. Rascher, G. Mohammed, Remote Sens. Environ. 168, 420-436 (2015)

13. R. Gaulton, F.M. Danson, F.A. Ramirez, O. Gunawan, Remote Sens. Environ. 132, 32-39 (2013)

14. R. Houborg, M.F. McCabe, A. Cescatti, A.A. Gitelson, Int. J. Appl. Earth Obs. Geoinf. 3, 1-6 (2015)

15. E.M. Perry, J.R. Davenport, Comput. Electron. Agric. 59, 56-65 (2007)
16. M.F. Noomen, K.L. Smith, J.J. Colls, M.D. Steven, A.K. Skidmore, and F.D. Van Der Meer, Int. J. Remote Sens. 29, 5987-6008 (2008)

17. F. Van Der Meer, Int. J. Appl. Earth Obs. Geoinf. 5, 55-68 (2004)

18. R.F. Kokaly, D.G. Despain, R.N. Clark, and K.E. Livo, Integrated Geoscience Studies in the Greater Yellowstone Area. Chapter N, 463-489 (2007) 